\title{
New and Newer Vaccines for SARS-CoV-2 Variants. Are the Major Vaccine Developers on the Right Track, Or Is Delipidation the Answer?
}

\author{
B. E. Cham \\ Australasian Institute of Medical Research, Port Vila, Republic of Vanuatu \\ Email: bill.cham@gmail.com
}

How to cite this paper: Cham, B.E. (2021) New and Newer Vaccines for SARS-CoV-2 Variants. Are the Major Vaccine Developers on the Right Track, Or Is Delipidation the Answer? Advances in Infectious Diseases, 11, 165-170.

https://doi.org/10.4236/aid.2021.112016

Received: March 27, 2021

Accepted: April 26, 2021

Published: April 29, 2021

Copyright $\odot 2021$ by author(s) and Scientific Research Publishing Inc. This work is licensed under the Creative Commons Attribution International License (CC BY 4.0).

http://creativecommons.org/licenses/by/4.0/

\begin{abstract}
Background: Global Covid-19 pandemic has led to remarkable scientific achievements resulting in the development and rapid implementation of vaccines towards the original wild-type SARS-CoV-2 virus. Most Covid-19 vaccines are targeted to only one protein (the Spike protein) on the virus. SARS-CoV-2 that causes Covid-19 naturally undergoes multiple mutations over time. Such mutations can be inconsequential or have dire consequences. The lack of effectiveness of current vaccines towards mutated variants of Covid-19 is of major concern. The objective of this study is to describe an optimal solvent system that creates, via delipidation, a non-synthetic, host-derived or nonhost-derived modified viral particle that has its lipid envelope removed, exposing hidden undenatured proteins from within the virus, that generate a positive immunologic response when administered into a host, thereby providing a vaccine that offers strong and broad protection against the virus. Methods: Lipid removal from viruses by specific procedures renders the exposure of hidden proteins. Protection by antibodies to all of the virus' protein types has shown to be far superior to protection by antibodies that are created by a single protein type. Results: Published studies with the Hepatitis virus, Pestivirus and HIV virus have reported the wide range of applications with this delipidation approach resulting in effectively long-term and broad protection vaccines. Conclusion: Mutations are rendering existing vaccines less effective. New approaches to obtain a more permanent vaccine that minimizes the effects of mutation are obtainable by delipidation of the viral particle and thereby creating vaccines that are more permanent with broad protection.
\end{abstract}

\section{Keywords}

SARS-CoV-2, Covid-19, Spike Protein, Variant, Mutation, Vaccine, Virus, 
Butanol, Diisopropylether, Delipidation, Total Protein

\section{Introduction}

Vaccine developers have done an amazing job to rapidly develop vaccines for the wild-type Covid-19. Although this is a great achievement, let us not accept complete victory with this microscopic parasite. This contagion is harder to defeat than you think. Nature is on its side by bestowing natural survival strategies that allow multiple mutations to overcome its attackers. Viruses, including SARS-CoV-2 coronavirus that causes Covid-19 constantly mutate. Such mutations can be inconsequential or, have dire consequences.

The current weapons (vaccines) to destroy the SARS-CoV-2 although effective towards the wild-type virus are old-fashioned in terms of their arsenal. Vaccine developers realize this and are clearly taking this threat seriously. Updated versions of the existing Covid-19 vaccines are already being considered by vaccine makers and governments. They are now admitting a similar cat and mouse game currently being applied as the requirement of yearly boosters of the flu vaccine.

The limitations of most current Covid-19 virus vaccines are attributed to affecting only the spike protein of the virus. This spike protein on the virus is essential for it to enter the cells where replication of the virus occurs. So, by blocking entry of the virus, no more viruses can be replicated. If a person has antibodies that can recognise the spike protein then this stops the virus from further activities. The three most advanced vaccines all work by getting our own cells under the direction of the viral mRNA to make copies of the virus spike protein. When our own cells make the spike protein, our immune response recognises it as foreign and makes humoral antibodies and $\mathrm{T}$ cells that specifically target the spike protein.

This is a very good approach to rapidly combat Covid-19, but what if the spike protein on the virus mutates to create a variant? The consequential outcome depends on the extent of the mutation of the protein. An optimum antibody recognises an antigen of a specific peptide that has to be in the "correct" functional conformation and orientation. Depending on the extent of the mutation of the spike protein, the antibody vaccine may still be completely effective, partial effective, or not effective at all!

\section{Methods}

Currently there are five Covid-19 variants of concern. The variant B.1.1.7 first detected in the United Kingdom; P.1 first detected in Japan/Brazil; B.1.351 first detected in South Africa; B.1.427 first detected in USA-California and B.1.429 first detected in USA-California. Mutations leading to these variants are seriously worrisome problems. It is highly likely that there will be more variants with hitherto unknown sequelae of the infection, before Covid-19 pandemic is over. 
The SARS-CoV-2 virus is much more complicated than just a spike protein. There are in fact four different types of proteins that form the overall structure of the virus particle: spike (S) proteins, envelope (E) proteins, membrane (M) proteins and nucleocapsid $(\mathrm{N})$ proteins. In a natural infection, our immune system recognises all these proteins as foreign to varying degrees.

SARS-CoV-2 is an enveloped virus that is surrounded by a lipid bilayer that supports its structural foundation. Since viruses are non-metabolic, they only reproduce within living host cells. The virus codes the proteins of the viral particle while the host cell codes the lipids and carbohydrates. Accordingly, the enveloped viral particles partially adopt the identity of the host cell via lipid and carbohydrate content, and are able to conceal the protein antigens associated with them, which would normally have initiated an immune response. Instead, the viral particle confuses the host immune system by presenting it with an antigenic complex that contains components of host tissues, and is perceived by the host immune system as partly "self" and partly "foreign".

Consequently, the immune system is forced to produce the "compromised" outcomes, which are ineffective antibodies to unexposed antigens "hidden" within the viral particle.

If these "hidden" protein antigens in the virus were to be exposed, then the immune system would recognise all of them as foreign and create antibodies to neutralise them, resulting in a polyvalent vaccine development. Polyvalent vaccines characteristically elicit both cellular and humoral responses and it is thought that collaboration between them is the key characteristic of efficacy. One essential requirement is to obtain the "hidden" proteins in their native undamaged forms.

\section{Results}

A similar technical problem was addressed in blood product research by the University of Queensland, where the aim of lowering the lipid concentration of plasma while preserving the biological activity, including enzymatic functions of plasma was met by the use of a specific solvent extraction system of either an ether such as diisopropylether (DIPE) or a mixture of an ether and an alcohol such as DIPE and butanol. Delipidation of serum with this organic solvent system causes dissociation of lipids (which are transferred into the organic solvent) and apolipoproteins, which remain soluble in the serum. Removal of lipids from their carrier lipoproteins does not affect their physiological roles, and does not affect lipid related enzyme activities [1] [2] [3] [4]. The epitopes of the delipidated proteins obtained using DIPE-butanol system are not affected by delipidation and indeed, it has been shown that the epitopes as measured by antigen-antibody interaction increases in such lipid-protein complexes after delipidation, suggesting that the delipidation process may lead to more efficient processing of lipid-associated proteins. Indeed, this technology was subsequently used to achieve regression of atherosclerosis, a main contributor of heart failure [5] [6] [7] [8]. 
Up until then, the major limiting step was obtaining stable structural, biochemical, and physiological active proteins after lipids were removed from their parent lipid-associated particles.

\section{Discussion}

Our group recognised the importance of possibly applying these observations to a viral platform. Having available a procedure that could gently remove lipids from lipid-associated particles such as viruses without harming the activity of the resultant proteins had not previously been explored. Such a procedure would allow, when applied as a vaccine, protection against all the antigenic proteins (S, E, M, N) of the virus and not only the Spike protein.

It was considered that, by delipidation of the viral lipid envelope and inactivating the virus, yet retaining all of the viral antigens would have a major benefit for therapeutic vaccination. Multi-antigen vaccines, combining both structural and non-structural antigens, aim to induce multi-specific immune response involving both the humoral and the cell mediated arms. A vigorous immune response to multiple targets maximises the control of the infection on the one hand while minimising the emergence of escape of viral mutants on the other hand.

This hypothesis was shown to actually be the case. Indeed, using this virus delipidation technology, vaccines were developed from certain lipid-containing viruses by removing the lipid envelope and exposing the protein antigens previously associated with the lipids and hidden beneath the lipid envelope.

This approach was applied by researchers at the Universities of Queensland and Sydney to the delipidation of a hepadna virus in a duck model. Delipidation of the virus resulted in the complete inactivation of the virus and the viral delipidated proteins retained epitopes that were capable of generating protective immunity and specific anti-hepatitis antibody in recipients. Only nanogram amounts of delipidated duck hepatitis B virus were required to produce a safe and effective vaccine. This suggests that the viral antigens in this system were preserved or even enhanced by the delipidation process [9].

These observations were confirmed by further studies at the University of Queensland and the Elizabeth Macarthur Agricultural Institute in New South Wales, who reported that delipidation of a Pestivirus; Bovine Viral Diarrhea virus in large animals resulted in viral inactivation and an effective safe vaccine for this virus was described [10].

Further studies with the SIV and HIV viruses confirmed that delipidation resulted in enhanced cell-mediated immune responses while reducing their infectiveness. It was also shown that delipidated viruses had negligible loss in viral proteins and that delipidated SIV viruses gave rise to an improved immunological response across a broader array of antigens when compared to non-delipidated viral particles.

Moreover, boosted delipidated HIV virus gave much higher antibody titers compared to the live HIV boosted monkeys [11]. 
Table 1. Evolution of the delipidation system.

\begin{tabular}{lc}
\hline & Reference \\
\hline Development of the delipidation procedure & {$[1]$} \\
Physicochemical properties of lipoprotein by the delipidation procedure & {$[2]$} \\
Stability and conformational retention of proteins after delipidation & {$[3]$} \\
Increased immunological response of proteins after removal of their lipid by delipidation & {$[4]$} \\
Plasma delipidation induces rapid regression of atherosclerosis in animals & {$[5]$} \\
Delipidation enhances reverse cholesterol transport in humans & {$[6]$} \\
Delipidation causes regression of atherosclerosis in non-primates, monkeys and humans & {$[8]$} \\
Mechanism of action by plasma delipidation resulting in regression of atherosclerosis & {$[9]$} \\
Delipidation of hepadnavirus resulting in viral inactivation and vaccine development & {$[10]$} \\
Delipidation of pestivirus resulting in viral inactivation and vaccine development & \\
Delipidation of SIV and HIV increases breath and strength of the overall mediated immune response in mice and monkeys
\end{tabular}

Table 1 shows the evolution of the delipidation system, its applications to the treatment of atherosclerosis and the inactivation of lipid-associated viruses with subsequent vaccine developments.

Using this delipidation platform it is possible to create combination vaccines of modified viral particles obtained from one or more strains of the virus and/or one or more types of virus, rendering a very wide scope of applications.

These observations clearly indicate that delipidation of lipid-containing viruses such as our current nemesis SARS-CoV-2 and its various mutated forms is a much wanted direction to explore and may be superior to the existing more single mutation-prone and repeatingly costly vaccines.

\section{Conclusions}

Current vaccines offer protection against the original wild-type Covid-19. New and newer variants of the SARS-CoV-2 virus are emerging frequently. In this regard it is extremely important to obtain unrestricted rapid worldwide vaccinations with currently available vaccines, this should limit and control the emergence of more variants. When a virus is widely circulating in a population and causing many infections, the likelihood of the virus mutating increases. The more opportunities a virus has to spread, the more it replicates and the more opportunities it has to undergo changes. Stopping the spread at the source remains key, we need to do everything possible to stop the spread of the virus in order to prevent mutations that may reduce the efficacy of existing vaccines.

There is evidence that mutations are rendering existing vaccines less effective or, in a worst-case situation, ineffective.

New approaches to obtain a more permanent vaccine that minimizes the effects of mutation must start now. Delipidation of lipid-associated viruses, including SARS-CoV-2, offers such a possibility and should be fully investigated. 


\section{Conflicts of Interest}

The author declares no conflicts of interest regarding the publication of this paper.

\section{References}

[1] Cham, B.E. and Knowles, B.R. (1976) A Solvent System for Delipidation of Plasma or Serum without Protein Precipitation. Journal of Lipid Research, 17, 176-181. https://doi.org/10.1016/S0022-2275(20)37003-6

[2] Cham, B.E. and Knowles, B.R. (1976) Changes in Electrophoretic Mobilities of Alpha and Beta Lipoproteins as a Result of Plasma Delipidation. Clinical Chemistry, 22, 305-309. https://doi.org/10.1093/clinchem/22.3.305

[3] Cham, B.E. and Knowles, B.R. (1976) In Vitro Partial Relipidation of Apolipoproteins in Plasma. Journal of Biological Chemistry, 251, 6367-6371. https://doi.org/10.1016/S0021-9258(20)81870-5

[4] Kostner, G.M., Avogaro, P., Bittolo Bon, G., Quinci, G.B. and Chinello, M. (1979) Determination of High Density Lipoproteins; Screening Methods Compared. Clinical Chemistry, 25, 939-942. https://doi.org/10.1093/clinchem/25.6.939

[5] Cham, B.E., Kostner, K.M., Shafey, T.M., Smith, J.L. and Colquhoun, D.M. (2005) Plasma Delipidation Process Induces Rapid Regression of Atherosclerosis and Mobilization of Adipose Tissue. Journal of Clinical Apheresis, 20, 143-153. https://doi.org/10.1002/jca.20060

[6] Sacks, F.M., Rudel, I.L., Conner, A., Keefe, A., Kostner, G., Baki, T., et al. (2009) Selective Delipidation of Plasma HDL Enhances Reverse Cholesterol Transport in Vivo. Journal of Lipid Research, 50, 894-907. https://doi.org/10.1194/jlr.M800622-JLR200

[7] Cham, B.E. and Chase, T.R. (2013) Intravascular Infusion of Autologous Delipidated Plasma Induces Antiatherogenic Lipoproteins and Causes Regression of Atherosclerosis: Studies in Non-Primates, Monkeys and Humans. Health, 5, 19-33. https://doi.org/10.4236/health.2013.57A1003

[8] Cham, B.E. (2015) Not All "Bad” Cholesterol Carriers Are Necessarily Bad and Not All "Good" Cholesterol Carriers Are as Good as Can Be: Plasma Delipidation, a Non-Pharmacological Treatment for Atherosclerosis. International Journal of Clinical Medicine 6, 690-699. https://doi.org/10.4236/ijcm.2015.69092

[9] Cham, B.E., Vickery, K., Tohidi-Esfahani, R. and Cossart, Y. (2006) Delipidation of a Hepadnavirus: Viral Inactivation and Vaccine Development. Journal of Virological Methods, 137, 160-163. https://doi.org/10.1016/j.jviromet.2006.05.027

[10] Cham, B.E., Shannon, A.D., Gu, X. and Elliot, A. (2007) Delipidation of a Pestivirus: Viral Inactivation and Vaccine Development in Large Animals. Research Journal of Biological Sciences, 2, 706-712.

[11] United States Patent US20050032222A1 (2004) Modified Viral Particles with Immunogenic Properties and Reduced Lipid Content Useful for Treating and Preventing Infectious Diseases. 